\title{
Comparison of the Immunogenicity of an Inactivated Classical Swine Fever Virus Vaccine Employing a Local Isolate With the Live Lapinized Classical Swine Fever Virus Vaccine
}

\author{
K.H. Steffi Rajakumar ${ }^{1 *}$, A. Ramesh ${ }^{2}$, K.G. Tirumurugaan ${ }^{2}$ and K. Kumanan ${ }^{3}$ \\ ${ }^{1}$ Department of Veterinary Microbiology, MVC, Chennai-7, Tamil Nadu, India \\ ${ }^{2}$ Vaccine Research Centre-Viral Vaccines, Centre for Animal Health Studies, TANUVAS, \\ Chennai-51, Tamil Nadu, India \\ ${ }^{3}$ Department of Animal Biotechnology, MVC, TANUVAS, Chennai-7; 4- MVC, TANUVAS, \\ Chennai-51, Tamil Nadu, India \\ *Corresponding author
}

\section{Keywords}

Inactivated adjuvanted CSF vaccine, ELISA, Immunogenicity

Article Info

Accepted: 06 September 2018 Available Online: 10 October 2018

\section{A B S T R A C T}

A study was conducted for assessing the immunogenicity of the inactivated PK-15 cell adapted classical swine fever virus (CSFV) vaccine in pigs under farm conditions. Thirty numbers of pigs of $10-12$ weeks of age maintained at two farms each were vaccinated. The 30 number of pigs from each farms were divided into three groups comprising of 10 animals each. Group I was vaccinated with the inactivated PK-15 cell adapted CSF virus vaccine adjuvanated with single (Oil-in-water O/W) emulsion. Group II was vaccinated with the inactivated PK-15 cell adapted CSF virus vaccine adjuvanated with double (water-in-oil-in-water $\mathrm{W} / \mathrm{O} / \mathrm{W}$ ) emulsion. Group III was vaccinated with the lapinized live CSFV vaccine. In addition, Group IV and V comprising 6 pigs each in farm located at Madhavaram milk colony, Chennai 51 were vaccinated with a booster dose with two types of inactivated PK-15 cell adapted CSFV vaccine with single and double emulsion adjuvant respectively on $28^{\text {th }}$ day after primary vaccination. With regard to humoral immune responses, the inactivated CSFV vaccine emulsified with single emulsion adjuvant generated mean humoral immune response lower than the threshold serum antibody levels (PI of 50\%) in the ELISA assay at $28 \mathrm{dpv}$ and comparable immune responses on 90 and 180 dpvwere observed in comparison with the lapinized live CSFV vaccine. However, the single emulsion vaccine was found effective in inducing humoral immune response in the absence of maternal antibodies. Farm trials of the inactivated CSFV vaccine with single emulsion adjuvant could generate and sustain the serum CSFV antibody levels until 180 dpv which is a major requirement under field conditions since the pigs are marketed at 8-9 months of age.

\section{Introduction}

Classical swine fever (CSF) is a highly contagious, haemorrhagic viral disease of pigs that causes huge economic loss to the pig industry due to high morbidity and mortality. CSF belongs to Pestivirus genus of the family Flaviviridae and the disease in pigs is 
characterised by fever, huddling, weakness, drowsiness, anorexia, conjunctivitis and constipation followed by diarrhoea. The disease remains widespread in India and in many parts of the world (Patil et al., 2012). Live attenuated CSF vaccines have been used for controlling the disease in pigs, the most common being the CSFV - C strain vaccine (Wilke et al., 2004). In India, a live attenuated lapinized CSF vaccine has been used as a prophylactic measure to control CSF in pigs with only few Institute of State Veterinary Biologicals in India producing limited doses of the vaccine. However, this production is not sufficient to cover even one percent of the total pig population (Nath et al., 2016) which is 10.29 million as per 19th livestock census. The efficacy of inactivated vaccine could be improved by incorporating adjuvants in the vaccine formulations (Tang et al., 2014; Awate et al., 2013). The selection and use of different type of adjuvants in the preparation of inactivated CSFV vaccine formulation is an important aspect in terms of development of both early and long lasting immunity (Coffman et al., 2010). The oil-in-water $(\mathrm{O} / \mathrm{W}$ - single emulsion) adjuvants induces a strong, short term immunity and found useful in case of disease outbreak conditions. Mosca et al., (2008) have demonstrated that $\mathrm{O} / \mathrm{W}$ emulsion adjuvants (MF59) was a strong modulator of "adjuvant core response genes" at the site of injection by upregulation of cytokines and chemokines. The water-in-oilin-water (W/O/W - double emulsion) induces an early and long lasting protection and promotes both Th1 cell response and IGg2 production (Shahiwala et al., 2008).

An inactivated PK-15 cell adapted CSF virus vaccine using local CSFV strain has been developed at Vaccine Research Centre - Viral Vaccines, CAHS, TANUVAS, Chennai - 51. The laboratory trials have indicated that the above said vaccine elicited sufficient immunity for up to six months and with stood the virulent CSF virus challenge in vaccinated pigs. Hence the present study is aimed at assessing the immune responses of the inactivated PK-15 cell adapted CSFV vaccines prepared with two types of adjuvants viz. single and double emulsion adjuvants under farm conditions. Different types of adjuvants are used in the preparation vaccine formulation to enable better and longer immunity in pigs. This study will be useful in understanding the performance of the above said inactivated CSF vaccines under field conditions and the results to be obtained in this study will help in large scale use of inactivated CSFV vaccine in the field which in turn will be beneficial to the pig farming community in controlling CSF.

\section{Materials and Methods}

\section{CSF vaccines and animals used}

The lapinized live CSFV vaccine obtained from State Institute of Veterinary Biologicals, Mhow, Madhya Pradesh was used as a standard. The inactivated PK-15 cell adapted CSFV vaccines developed with a local characterized CSFV strain (Vengaivasal strain) adjuvanated with a single emulsion adjuvant and a double emulsion adjuvant were used (developed at Vaccine Research CentreViral Vaccines, Centre for Animal Health studies, TANUVAS).

Pigs at Institutional Livestock Farm Complex (ILFC), Madhavaram Milk colony and at Post graduation research institute in animal sciences (PGRIAS), of TANUVAS were vaccinated for this study. Blood samples were collected in vacutainer tubes and used for this study. Serum was separated from the blood collected in plain vacutainer tubes and stored at $-80 \mathrm{oC}$ until use. Group I was vaccinated with the inactivated PK-15 cell adapted CSF virus vaccine adjuvanated with single (Oil-inwater $\mathrm{O} / \mathrm{W}$ ) emulsion. Group II was 
vaccinated with the inactivated PK-15 cell adapted CSF virus vaccine adjuvanated with double (water-in-oil-in- water W/O/W) emulsion. Group III was vaccinated with the lapinized live CSFV vaccine. Both the adjuvants were provided by Seppic, France. In addition, Group IB and IIB comprising 6 pigs each in farm located at Madhavaram milk colony, Chennai 51 were vaccinated with a booster dose with two types of inactivated PK15 cell adapted CSFV vaccine with single and double emulsion adjuvant respectively on 28th day after primary vaccination.

\section{ELISA for assessing humoral response in vaccinated pigs}

The humoral immune responses in pigs to CSFV vaccination were assessed by detecting the serum CSFV antibodies levels by ELISA assay using PrioCHECK ${ }^{\circledR}$ CSFV antibody ELISA system according to the manufacturer's instructions. The assay provides a threshold value of percent inhibition PI\% of 50\%. Any value above 50\% is termed as positive. The mean OD450 value of all reference serum was calculated and the corrected OD was also measured.

Percent Inhibition $(\mathrm{PI})=($ Corrected OD450 test sample/ Corrected OD450 reference serum4) x100

Interpretation of the percent inhibition PI vaues:

PI $=<30 \%$ Negative; PI $=31-50 \%$ Inconclusive; $\mathrm{PI}=>50 \%$ positive

\section{Results and Discussion}

Inactivated CSFV vaccine with single emulsion vaccine (Group I vs III)

The mean humoral immune response was lower (PI of 42\%) than the threshold value (PI of $50 \%$ ) in the inactivated CSFV vaccine with single emulsion adjuvant group at $28 \mathrm{dpv}$ (Table 1). However at the same point of time, the conventional lapinized live CSFV vaccine elicited a mean HIR percent inhibition value of $94.5 \%$ (Table 3). The mean humoral immune response at $90 \mathrm{dpv}$ and $180 \mathrm{dpv}$ in the inactivated CSFV vaccine with single emulsion adjuvant group (PI of 95\% and 75\%) was equivalent to that of lapinized live CSFV vaccine group (PI of $78 \%$ and $77 \%$ ) (Figure 1).

Inactivated CSFV vaccine with double emulsion vaccine (Group II vs III)

The mean humoral immune response was higher (PI of 75.66\%) than the threshold value in the inactivated CSFV vaccine with double emulsion adjuvant group at 28dpv (Table 2). However at the same point of time, the conventional lapinized live CSFV vaccine elicited a mean value of PI of $94.51 \%$ (Table $3)$. The mean humoral immune response at 90 dpv in the inactivated CSFV vaccine with double emulsion adjuvant group was just at threshold value (PI of 53.26\%) and fell lower than the threshold value at $180 \mathrm{dpv}$ (PI of $47.5 \%$ ) whereas the conventional lapinized live CSFV vaccine were higher than the threshold value throughout the study period of 180 days (PI of $78.6 \%$ and $77.6 \%$ at $90 \mathrm{dpv}$ and 180dpv respectively) (Figure 2).

\section{Booster vaccination in single emulsion adjuvant vaccine group (Group IB vs III)}

The mean humoral immune response was higher (PI of 69\%)than the threshold value in the booster vaccination group vaccinated with inactivated CSFV vaccine with single emulsion adjuvant on 28dpv and PI of $99.8 \%$ on $90 \mathrm{dpv}$ (Table 4) which was higher than the mean antibody response of the lapinized live CSFV vaccine (PI of 78.7\%) (Table 3). However, the mean HIR was comparable as 
that of lapinized live CSFV vaccine on 180 dpv (Figure 3).

Booster vaccination in double emulsion vaccine group (Group IIB vs III)

The mean humoral immune response in the booster vaccination group vaccinated with inactivated CSFV vaccine with double emulsion adjuvant was higher than the threshold value at all study point of time with a PI of $86 . \% ; 70 \%$ and $54.2 \%$ at $28 \mathrm{dpv}, 90 \mathrm{dpv}$ and $180 \mathrm{dpv}$ respectively (Table 5). However the conventional lapinized live CSFV vaccine showed higher mean HIR at $180 \mathrm{dpv}$ (PI of $77.6 \%$ ) even though the other days showed not much difference on comparison with booster vaccination group (Figure 4).

Comparison of inactivated CSFV vaccine with single and double emulsion adjuvant

The mean humoral immune response was lower (PI of 42\%) than the threshold value (PI of $50 \%$ ) in the inactivated CSFV vaccine with single emulsion adjuvant group at 28dpv (Table 1). Whereas at the same point of time, pigs vaccinated in group II showed a higher mean HIR value of PI of $75.7 \%$ (Table 2). However, the mean antibody levels of group I was found to be increased (PI of 97.4\%) at 90 dpv whereas there was a fall of mean antibody levelsin group II vaccinated pigs at the same point of time (PI of 53.3\%). At $180 \mathrm{dpv}$, themean antibody levels were above the threshold value in group I (PI of 75.5\%) whereas it was below the threshold value in group II at the same point of time (PI of $47.5 \%$ ) (Figure 5).

Comparison of primary and booster vaccination with inactivated $C S F V$ vaccine with single emulsion adjuvant

The mean humoral immune response was lower (PI of 42\%) than the threshold value (PI of $50 \%$ ) in the inactivated CSFV vaccine with single emulsion adjuvant group at $28 \mathrm{dpv}$ (Table 1). While pigs in group IB given booster vaccine showed a higher mean antibody response (PI of $69 \%$ ) at the same point of time (Table 4). At $90 \mathrm{dpv}$ both groups showed higher mean antibody response (PI of $97.4 \%$ and $99.8 \%$ ) respectively. Group IB showed a comparable mean antibody response at $180 \mathrm{dpv}$ (PI of $77.8 \%$ ) as that of group I (PI of $75.53 \%$ ) (Figure 6).

Comparison of primary and booster vaccination with inactivated $C S F V$ vaccine with double emulsion adjuvant

Both group II and IIB showed a mean antibody levels more than the threshold value at $28 \mathrm{dpv}$ (group II- PI of $75.7 \%$, group IIB PI of $86.7 \%$ ). At $90 \mathrm{dpv}$, group IIB showed a higher mean antibody response (PI of 70\%) (Table 5) when compared to group II (PI of $53.26 \%$ ). However at $180 \mathrm{dpv}$, primary vaccination group II showed lower mean antibody response (PI of 47.5\%) when compared to booster vaccination group IIB (PI of $54.2 \%$ ) (Figure 7).

Comparison of booster vaccination with inactivated CSFV vaccine with single and double emulsion adjuvant

At $28 \mathrm{dpv}$, both the booster vaccination groups (IB and IIB) showed higher than the threshold mean antibody response (PI of $69 \%$ and $86.7 \%$ ). However at $90 \mathrm{dpv}$, group IB showed higher mean antibody response (PI of 99.8\%) (Table 4) than group IIB (PI of 70\%) (Table 5). At $180 \mathrm{dpv}$, the mean antibody levels in both group IB (PI of $77.8 \%$ ) and IIB (PI of $54.2 \%)$ were higher than the threshold value (Figure 8).

Vaccination is the efficient and effective way to control CSF in pigs. Currently lapinized live CSFV vaccine is being used in many 
countries to protect pigs against CSF (Liu et al., 2011). In India, a lapinized live CSFV vaccine has been used as a prophylactic measure to control CSF in pigs with only few State Veterinary Biologicals in India producing limited doses. However, this vaccine production is not sufficient to cover even one percent of the total pig population (Nath et al., 2016) which is 10.29 million as per 19thlivestock census. In addition, there is a problem of producing bulk doses of the lapinized CSFV vaccine at a time due to ethical issues of using live rabbits for vaccine production as well as the difficulty in standardizing the virus concentration for every batch being produced. In this context, a cell culture based CSFV vaccine would be a potential alternate (Nath et al., 2016).

Development of a cell culture based live vaccine requires long time to obtain a completely attenuated CSF seed virus and the same has to be subjected to many quality control regimens including back passage studies. Hence, preparation of an inactivated cell culture based CSFV vaccine by incorporating a characterized CSFV field isolate could be a potential safe alternate to meet out the immediate demand for the vaccine to control CSF in pigs.

Table.1 Mean serum ELISA antibody levels in pigs vaccinated with single emulsion inactivated CSFV vaccine (Group I)

\begin{tabular}{|c|c|c|c|c|c|c|c|c|}
\hline \multirow{2}{*}{$\begin{array}{c}\text { serum } \\
\text { Days of } \\
\text { Collection }\end{array}$} & \multicolumn{6}{|c|}{$\begin{array}{l}\text { Animal Number } \\
\text { antibody as PI\%) }\end{array}$} & \multirow[t]{2}{*}{$\begin{array}{l}\text { Mean } \\
\text { (PI \%) }\end{array}$} & \multirow[t]{2}{*}{$\mathrm{SE}$} \\
\hline & A1 & A2 & A3 & A4 & A5 & A6 & & \\
\hline 0 Day & 1.2 & 1.5 & -21.3 & -21.3 & 98 & 98 & 26 & 23.1375 \\
\hline $28 \mathrm{dpv}$ & 99.6 & 99.6 & 100 & 100 & -73 & -73 & 42.2 & 36.42951 \\
\hline O dpv & 100 & 100 & 99.7 & 99.7 & 92.39 & 92.39 & 97.4 & 1.57366 \\
\hline $180 \mathrm{dpv}$ & 100 & 100 & -46 & 100 & 99.6 & 99.6 & 75.5 & 24.3068 \\
\hline
\end{tabular}

Table.2 Mean serum ELISA antibody levels in pigs vaccinated with double emulsion inactivated CSFV vaccine (Group II)

\begin{tabular}{|c|c|c|c|c|c|c|c|c|}
\hline $\begin{array}{c}\text { Days of } \\
\text { serum } \\
\text { collection }\end{array}$ & \multicolumn{6}{|c|}{$\begin{array}{c}\text { Animal Number } \\
\text { (Serum ELISA antibody as PI\%) }\end{array}$} & $\begin{array}{c}\text { MIEAN } \\
(\text { PI \% ) }\end{array}$ & SE \\
\hline 0 Day & -55 & -55 & -15.0 & -15.0 & -66 & 25 & -30.2 & 14.16667 \\
\hline 28 dpv & 60 & 60 & 100 & 100 & 67 & 67 & 75.7 & 7.800285 \\
\hline 90 dpv & 52.37 & 52.37 & 52.37 & 52.37 & 56.28 & 53.8 & 53.3 & 0.64757 \\
\hline 180 dpv & 41.33 & 41.33 & 50.1 & 50.1 & 51.1 & 51.1 & 47.5 & 1.962797 \\
\hline
\end{tabular}


Table.3 Mean serum ELISA antibody levels in pigs vaccinated with lapinized liveCSFV vaccine (Group III)

\begin{tabular}{|c|c|c|c|c|c|c|c|c|}
\hline \multirow{2}{*}{$\begin{array}{l}\text { Days of } \\
\text { serum } \\
\text { collection }\end{array}$} & \multicolumn{6}{|c|}{$\begin{array}{c}\text { Animal Number } \\
\text { (Serum ELISA antibody as PI\%) }\end{array}$} & \multirow[t]{2}{*}{$\begin{array}{l}\text { MEAN } \\
(\text { PI \%) }\end{array}$} & \multirow[t]{2}{*}{ SE } \\
\hline & C1 & C2 & C3 & $\mathrm{C4}$ & C5 & C6 & & \\
\hline 0 Day & -37.0 & -67.0 & -39 & 1.6 & 1.6 & 1.6 & -23.0 & 11.83705 \\
\hline $28 \mathrm{dpv}$ & 99.6 & 99.6 & 75.4 & 99.4 & 99.4 & 93.67 & 94.5 & 3.939281 \\
\hline $90 \mathrm{dpv}$ & 96 & 96 & 80 & 80 & 60 & 60 & 78.7 & 6.586181 \\
\hline $180 \mathrm{dpv}$ & 79 & 79 & 84 & 84 & 70 & 70 & 77.7 & 2.590581 \\
\hline
\end{tabular}

Table.4 Mean serum ELISA antibody levels in booster vaccination group with single emulsion inactivated CSFV vaccine (Group IB)

\begin{tabular}{|c|c|c|c|c|c|c|c|c|}
\hline \multirow{2}{*}{$\begin{array}{c}\text { Days of } \\
\text { serum } \\
\text { collection }\end{array}$} & \multicolumn{6}{|c|}{$\begin{array}{c}\text { Animal Number } \\
\text { (Serum ELISA antibody as PI\%) }\end{array}$} & \multirow[t]{2}{*}{$\begin{array}{l}\text { MEAN } \\
(\text { PI \%) }\end{array}$} & \multirow[t]{2}{*}{ SE } \\
\hline & D1 & D2 & D3 & D4 & D5 & D6 & & \\
\hline 0 Day & 100.0 & 100.0 & 71.58 & 71.58 & - & - & 85.8 & 6.698658 \\
\hline $28 \mathrm{dpv}$ & 69.7 & 69.7 & 63.4 & 65.4 & 75.7 & 70 & 69.0 & 1.743448 \\
\hline $90 \mathrm{dpv}$ & 100 & 100 & - & - & 99.6 & 99.6 & 99.8 & 0.094281 \\
\hline $180 \mathrm{dpv}$ & - & - & 52 & 60 & 99.5 & 99.5 & 77.8 & 10.33938 \\
\hline
\end{tabular}

Table.5 Mean serum ELISA antibody levels in booster vaccination group with double emulsion inactivated CSFV vaccine (Group IIB)

\begin{tabular}{|c|c|c|c|c|c|c|c|c|}
\hline \multirow{2}{*}{$\begin{array}{l}\text { Days of } \\
\text { serum } \\
\text { collection }\end{array}$} & \multicolumn{6}{|c|}{$\begin{array}{c}\text { Animal Number } \\
\text { (Serum ELISA antibody as PI\%) }\end{array}$} & \multirow[t]{2}{*}{$\begin{array}{l}\text { MEAN } \\
\text { (PI \%) }\end{array}$} & \multirow[t]{2}{*}{ SE } \\
\hline & E1 & E2 & E3 & E4 & E5 & E6 & & \\
\hline 0 Day & -66.0 & 25.0 & - & - & - & - & -20.5 & 26.26944 \\
\hline $28 \mathrm{dpv}$ & 60 & 60 & 100 & 100 & 100 & 100 & 86.7 & 8.43274 \\
\hline $90 \mathrm{dpv}$ & 51.1 & 51.1 & 53.8 & 97 & 97 & - & 70.0 & 10.07236 \\
\hline $180 \mathrm{dpv}$ & 51.1 & 51.1 & -30 & 99.5 & 99.5 & - & 54.2 & 21.61503 \\
\hline
\end{tabular}


Fig.1 Comparison of humoral immune responses across inactivated CSFV vaccine with single emulsion adjuvant (Group I) and lapinized live CSFV vaccine (Group III)

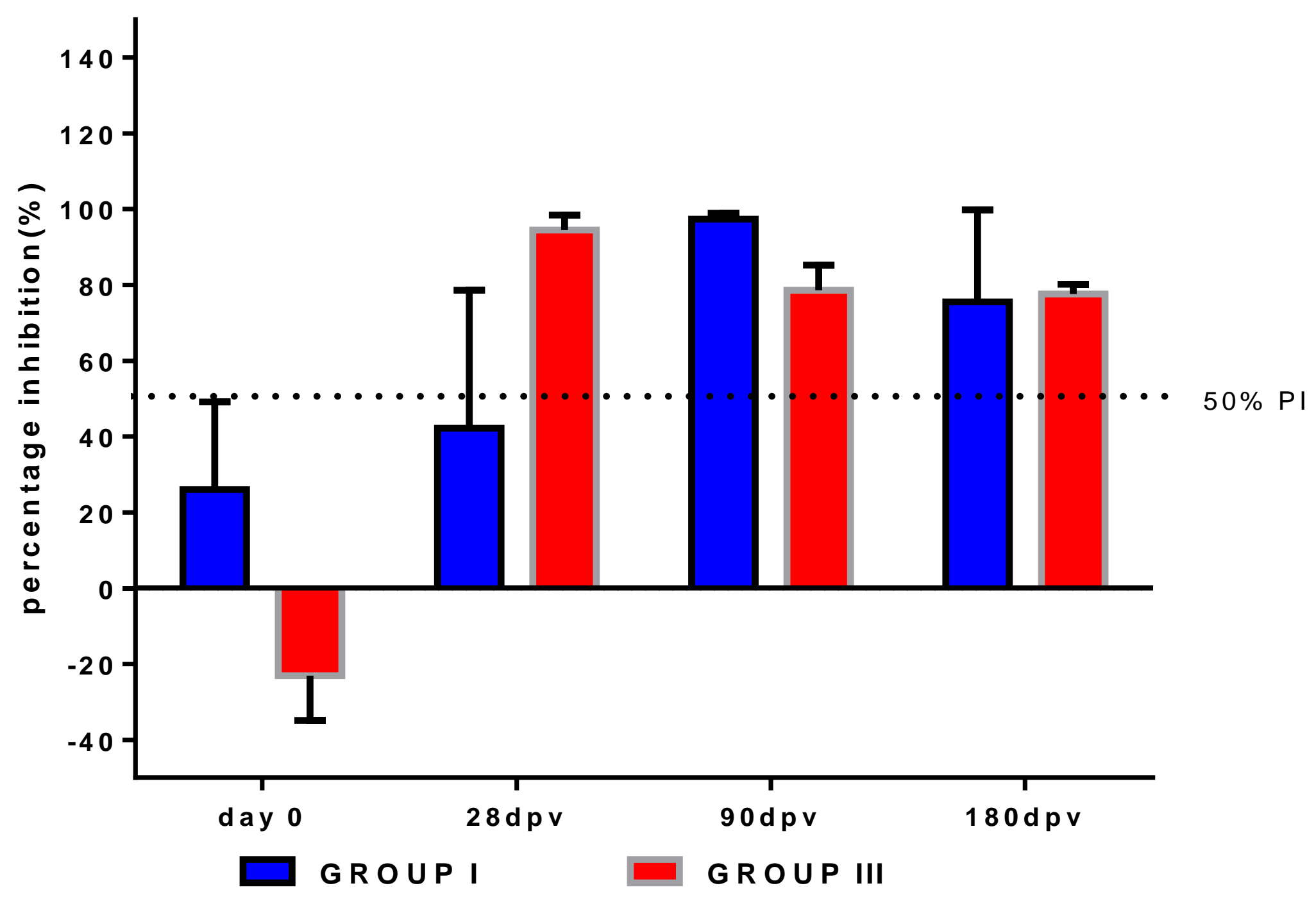


Fig.2 Comparison of humoral immune responses across inactivated CSFV vaccine with double emulsion adjuvant (Group II) and lapinized live CSFV vaccine (Group III)

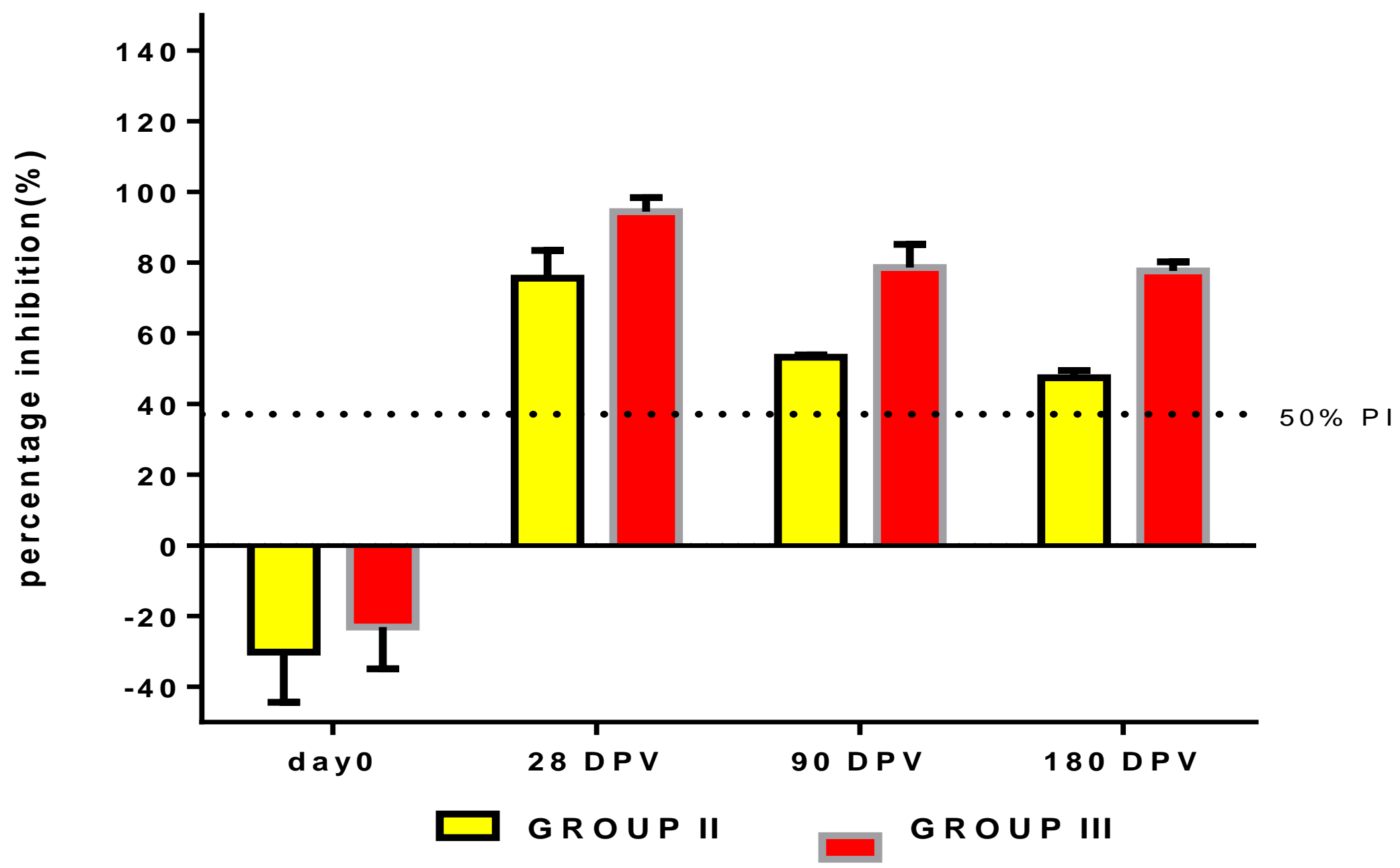


Fig.3 Comparison of humoral immune responses across booster vaccination with inactivated CSFV vaccine with single emulsion adjuvant (Group IB) and lapinized live CSFV vaccine (Group III)

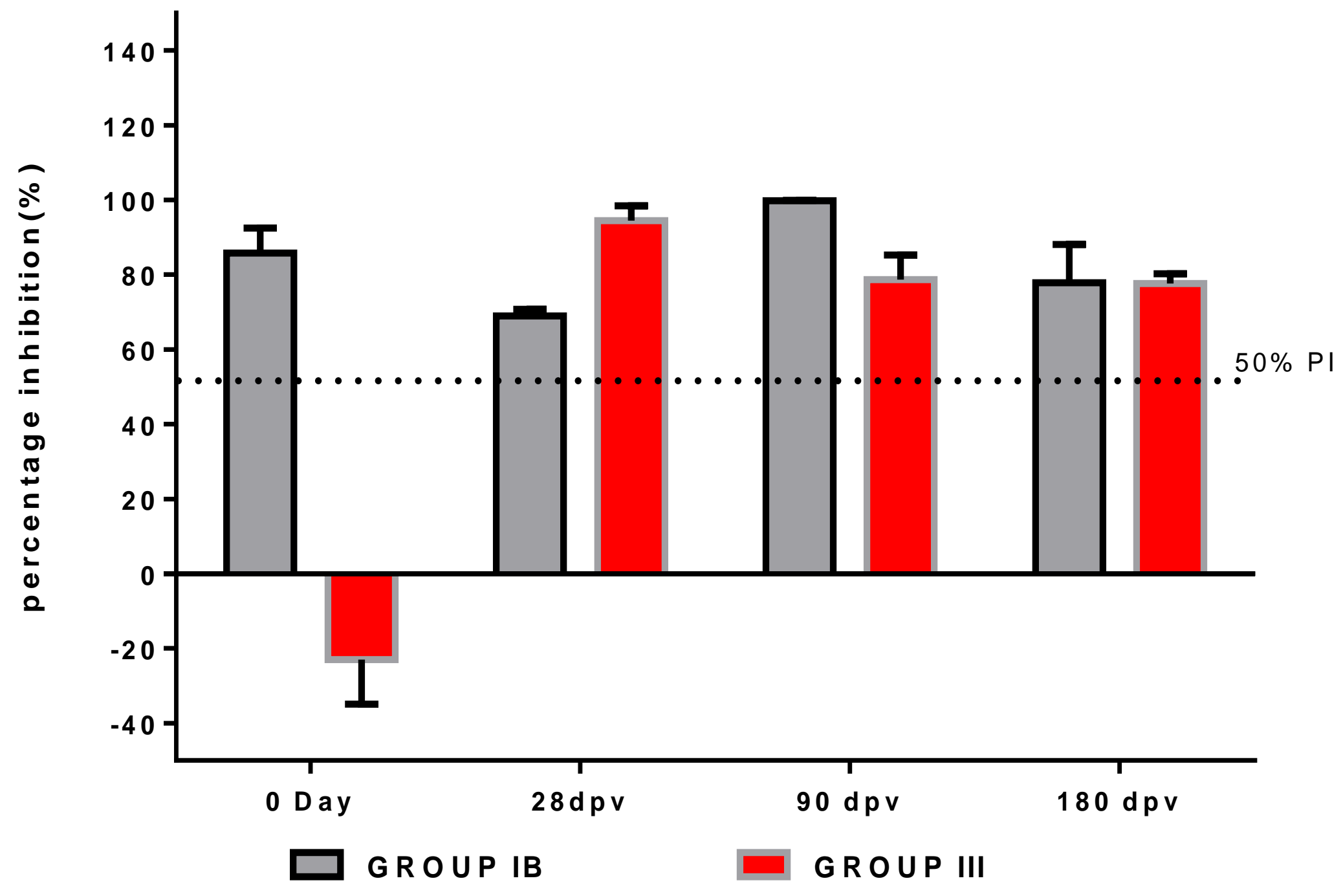


Fig.4 Comparison of humoral immune responses across booster vaccination with inactivated CSFV vaccine with double emulsion adjuvant (Group IIB) and lapinized live CSFV vaccine (Group III)

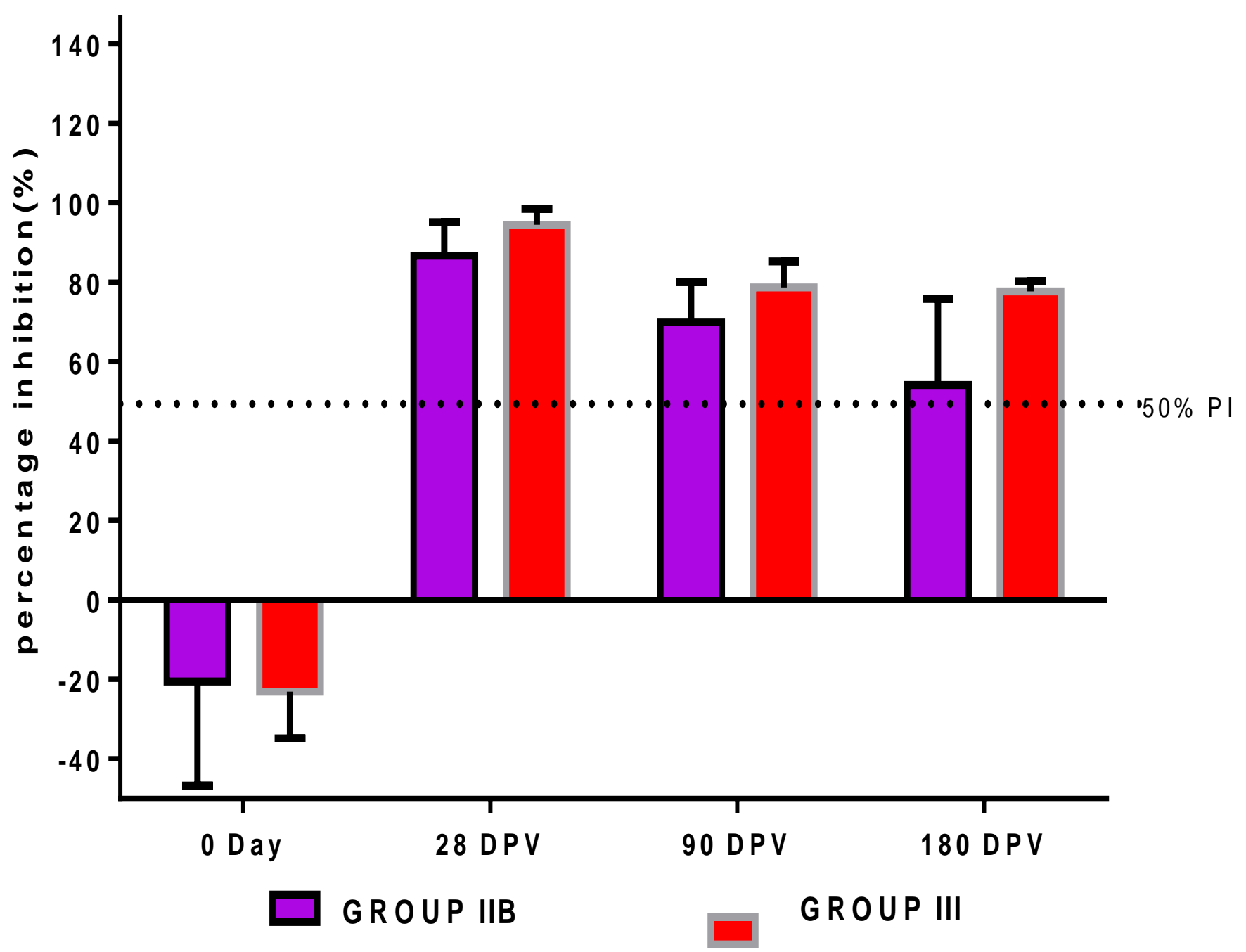


Fig.5 Comparison of humoral immune responses across inactivated CSFV vaccine with single and double emulsion adjuvants (Group I and II)

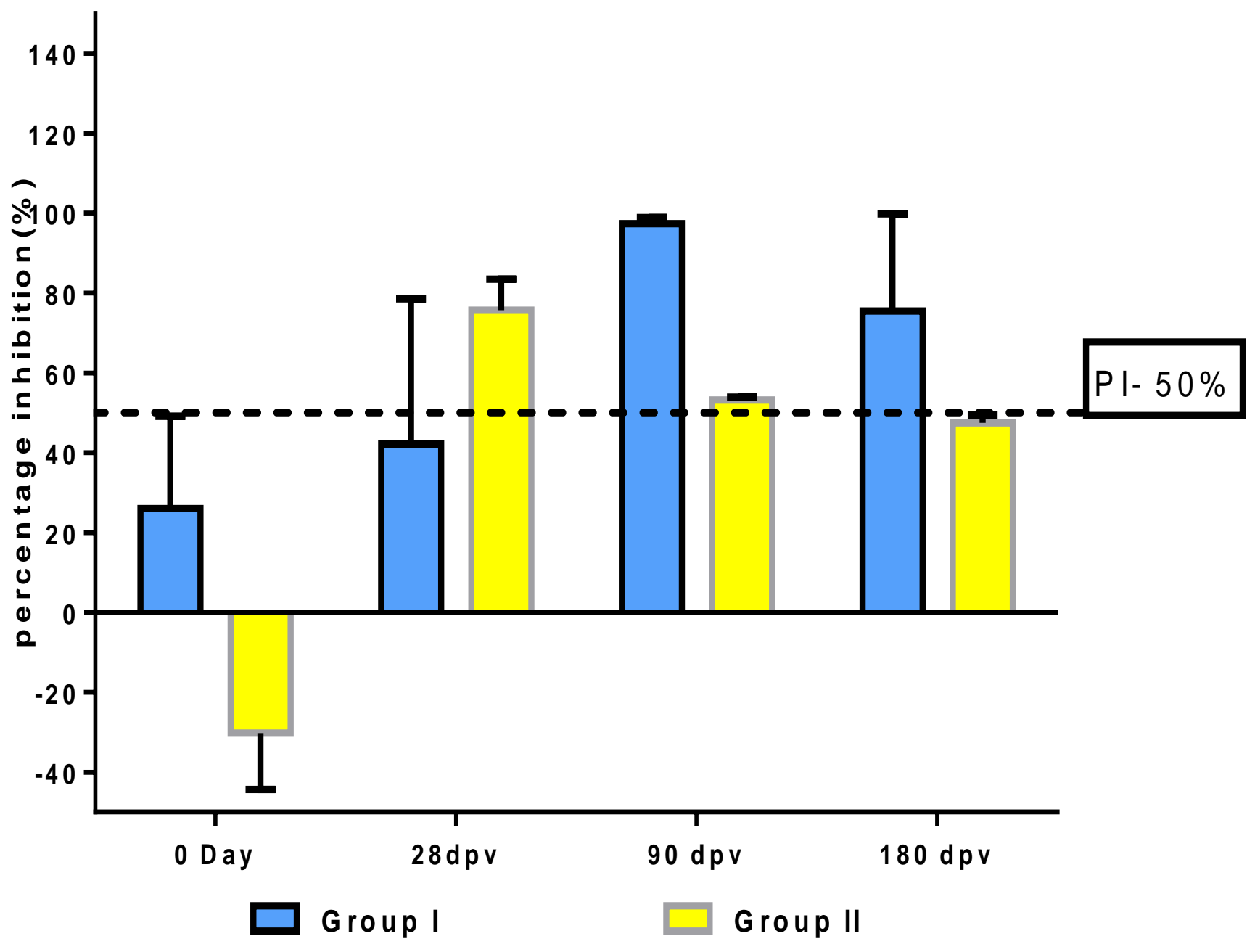


Fig.6 Comparison of humoral immune responses across primary and booster vaccination with inactivated CSFV vaccine with single emulsion adjuvants (Group I and IB)

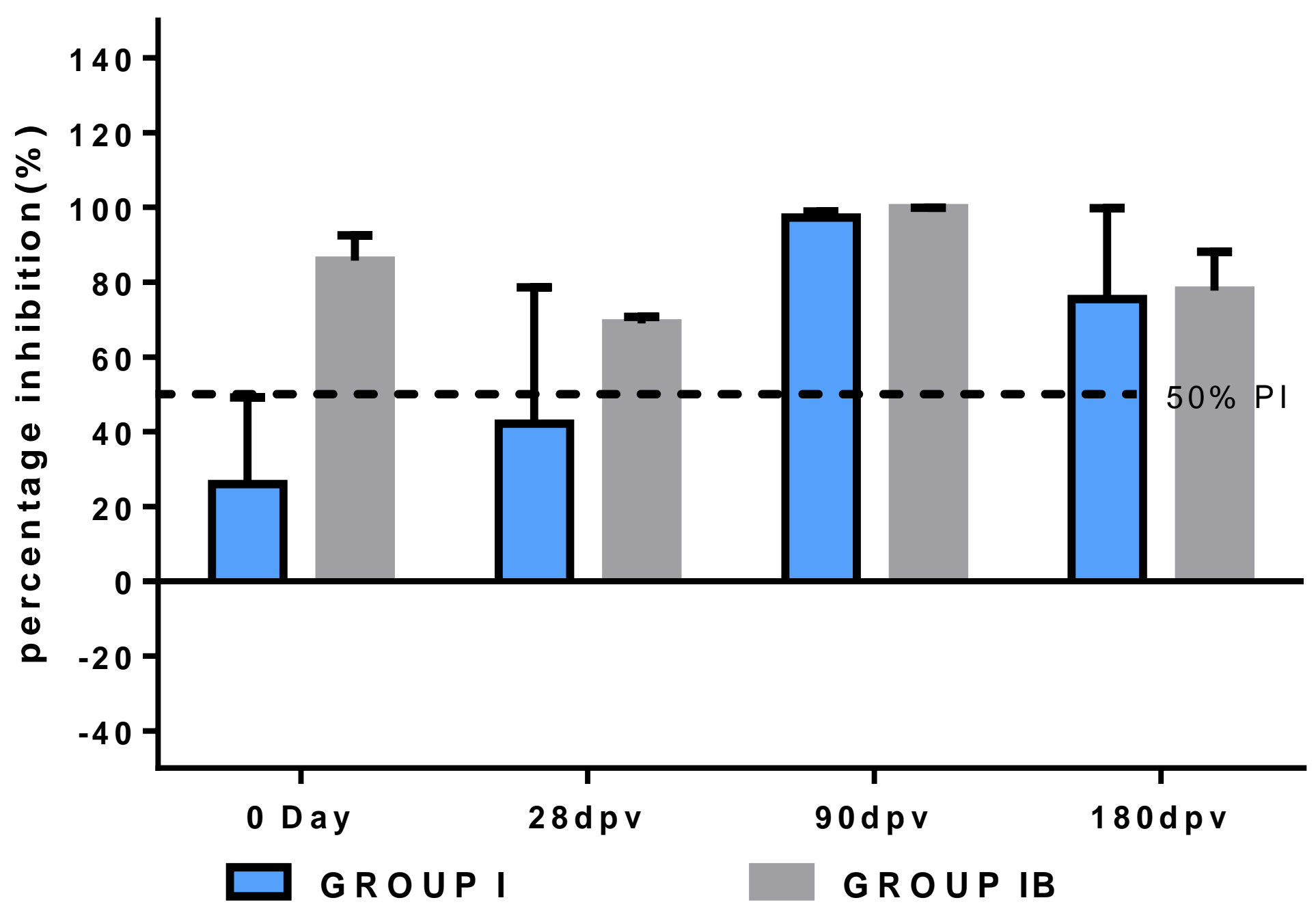


Fig.7 Comparison of humoral immune responses across primary and booster vaccination with inactivated CSFV vaccine with double emulsion adjuvants (Group I and IB)

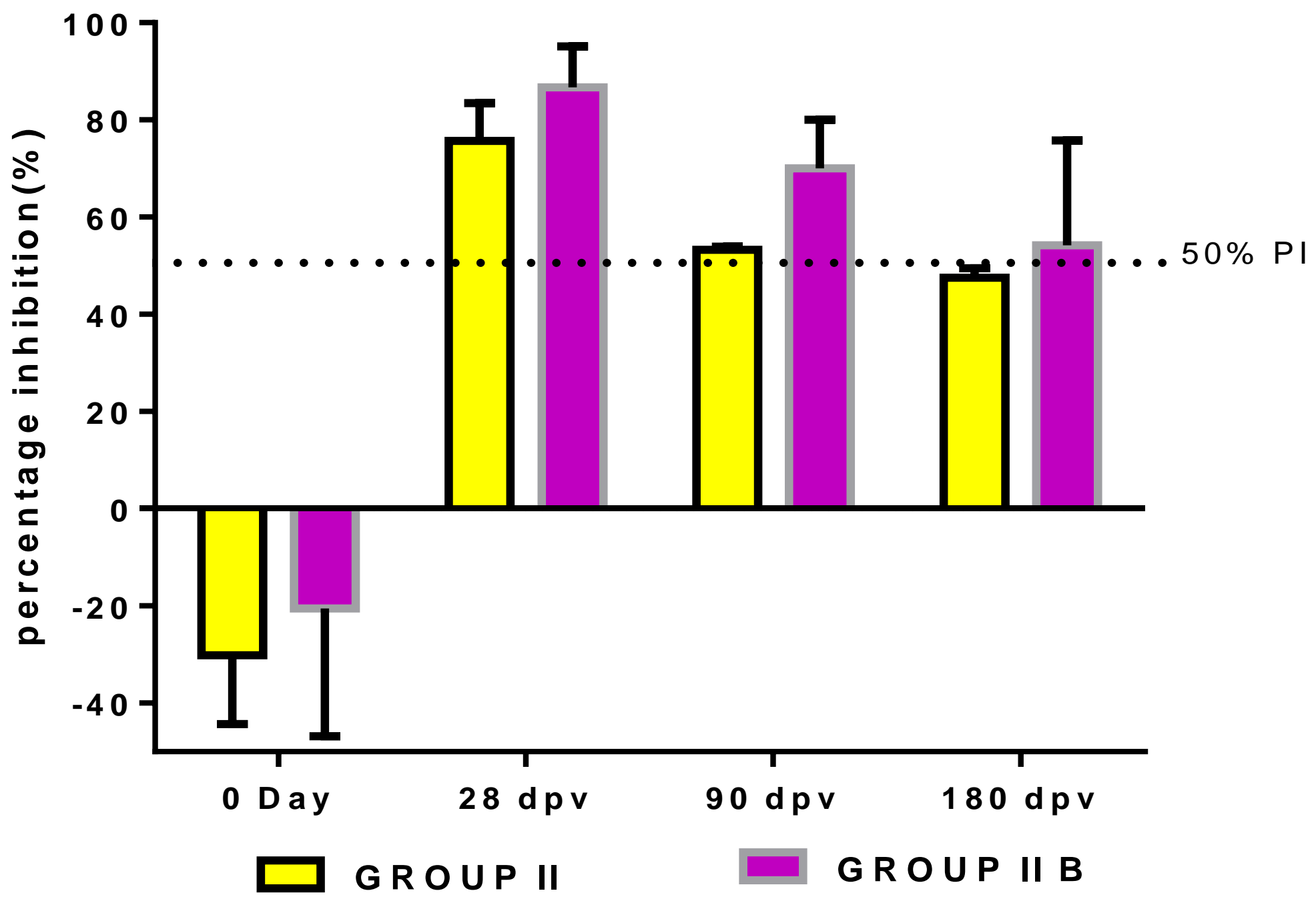


Fig.8 Comparison of humoral immune responses across booster vaccination with inactivated CSFV vaccine with single and double emulsion adjuvants (Group IB and IIB)

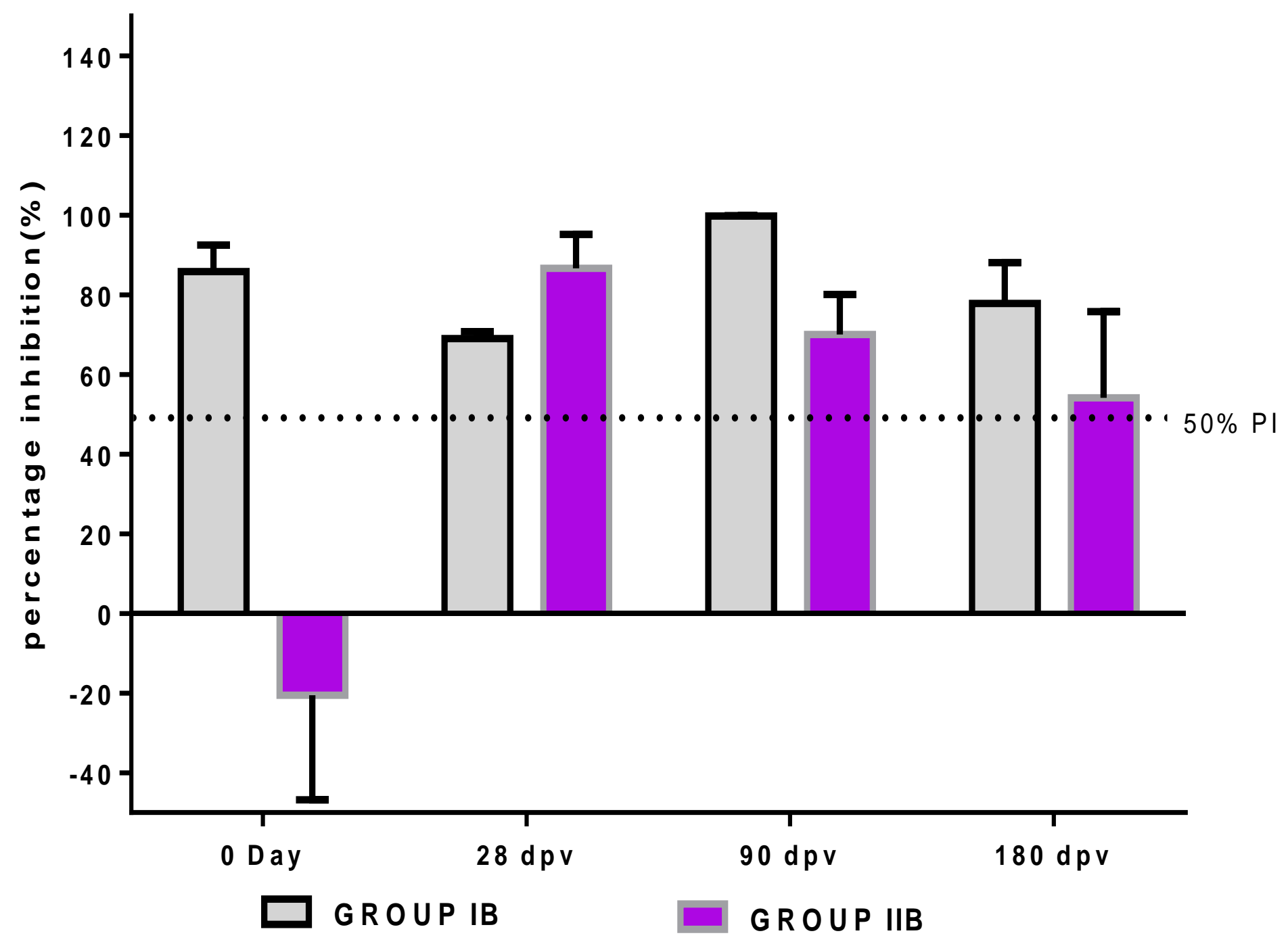


The efficacy of inactivated vaccine could be improved by incorporating adjuvants in the vaccine formulations (Tang et al., 2014; Awate et al., 2013).The selection and use of different type of adjuvants in the preparation of an inactivated CSFV vaccine formulation is an important aspect in terms of development of both early and long-lasting immunity (Coffman et al., 2010). The single emulsion oil-in-water adjuvants induced strong, short term immunity by up regulation of cytokines and chemokines and effective in case of disease outbreaks (Mosca et al., 2008). The double emulsion water-in-oil-inwater induced an early and long-lasting protection (Park et al., 2016) and promotes Th1 response and IgG production (Shahiwala et al., 2008). In the present study, no raise in body temperature was observed in pigs vaccinated with the inactivated CSFV vaccines prepared with both type of single and double emulsion adjuvants.

No adverse reactions at the site of vaccination and no untoward effects were observed in vaccinated pigs thus indicating the safety of the inactivated CSFV vaccines for use in pigs under farm conditions.

Vaccination with a C-strain lapinized live CSFV vaccine had induced neutralizing antibodies that usually appear about 2 weeks after vaccination and increased until at least 4- 12 weeks (Precausta et al., 1983; Terpstra et al., 1990; Dahle and Liess, 1995). Immune response to $\mathrm{C}$ - strain immunization has been found to persist for many years though some pigs had varied responses (Terpstra and Tielen, 1976). In the present study, the ability of inducing a good level of humoral immune response by two inactivated CSFV vaccines adjuvanated with two different adjuvants in pigs with varied levels of maternally derived CSFV antibodies under farm conditions were studied in comparison with lapinized live CSFV vaccine.
The inactivated PK-15 cell adapted CSFV vaccine adjuvanated with single emulsion adjuvant formulation was prepared at the antigen adjuvant ratio of 75:25 (3 parts: 1 part) as per manufacture's instruction with an antigen payload of $7 \log 10$ TCID50 per dose. The piglets were vaccinated at two and a half months to three months of age, irrespective of the maternal antibody levels at the time of vaccination. This single emulsion adjuvanated inactivated CSFV vaccine generated mean humoral immune response lower (PI of 42\%) than the threshold serum antibody levels (PI of $50 \%$ ) in the ELISA assay at 28 days post vaccination and comparable responses on 90 and $180 \mathrm{dpv}$ in comparison with the lapinized live CSFV vaccine (PI of 94.5\%) in the overall study. Earlier studies also showed the efficiency of single emulsion adjuvant in inducing and maintaining a humoral immune response to produce good short term immunity (Aucouturier et al., 2001). Thus the humoral immune responses generated in pigs with single emulsion vaccine could have been possible because of the optimum antigen payload and also the effective presentation and release of the antigen to host immune system.

This study being a field experimentation of the inactivated CSFV vaccine, we observed varied levels of maternally derived antibodies in some of the piglets at day 0 of vaccination across the different groups. We also observed MDA levels of PI of 98 to $100 \%$ in some of the piglets confirming the existence of maternal antibody at the time of vaccination. Similar observations were reported by Nath $e t$ $a l$. , (2016) that desirable level of MDA were observed for up to 42 days of age in piglets and the MDA levels reached a minimum level from about 10 weeks of age onwards and the MDA titres decreased to below the detection limit at an age of 84 days (Klinkenberg et al., 2002). However, in the absence of maternal antibodies, it is observed that the single 
emulsion vaccine was effective in inducing very high levels of mean humoral immune response at $28 \mathrm{dpv}$ (PI of 99.6-100\%). Farm trials of the inactivated CSFV vaccine with single emulsion adjuvant could generate and sustain the serum CSFV antibody levels until $180 \mathrm{dpv}$ which is a major requirement under field conditions since the pigs are marketed at 8-9 months of age.

The inactivated CSFV vaccine adjuvanated with double emulsion adjuvant formulation was prepared at the antigen adjuvant ratio of 50:50 (1 parts: 1 part) as per manufacture's instruction with an antigen payload of $7 \log 10$ TCID50 per dose. In this group, the mean serum CSFV antibody response was higher than the threshold level (PI of 50\%) at $28 \mathrm{dpv}$ and could be maintained until 90 days post vaccination. However, the antibody response decreased to below the threshold value of PI of $50 \%$ at $180 \mathrm{dpv}$ in comparison with lapinized live CSFV vaccinated group.

The double emulsion adjuvant is capable of eliciting an early immunity as indicated by a good level of antibody response in the absence of maternal antibody at 28 days post vaccination and maintain antibody threshold levels only up to 90 days post vaccination indicating a need for booster vaccination at 90 days to maintain the serum antibody level up to market age.

In this study, even though we maintained equal levels of antigen payload in terms of viral titre irrespective of the adjuvants in the vaccine formulation, it is found that the single adjuvanated inactivated CSFV vaccine performs better in not only eliciting higher threshold levels of antibody responses at 28 days post vaccination in the absence of maternal antibodies which is comparable to that of commonly used lapinized live CSFV vaccine but also helps in sustaining the levels until 180 dpv. Since the double emulsion vaccine could sustain threshold antibody levels until 90dpv, we explored the option of including a booster vaccination in both single and double emulsion vaccinated groups at 28 days post primary vaccination. In the case of the single emulsion vaccinated group there was no improvement in the threshold levels of antibody until $180 \mathrm{dpv}$ however, in the case of the double emulsion vaccinated group, a booster vaccination at 28 dpv could maintain the threshold serum antibody levels (above PI of 50\%) until 180 days post vaccination. However, earlier studies reported that the Montanide 201VG is a highly efficient adjuvant used to enhance both the cell mediated immune response and humoral immune responses with FMD vaccine and inactivated blue tongue vaccine (Sathyanarayanan, 2017).

Comparing the two adjuvanated inactivated CSFV vaccines tested in this study, the single emulsion vaccine generated better humoral immune responses early post vaccination which could be sustained until 180 days (period of study). With the double emulsion vaccine, even through the threshold levels of serum CSFV antibodies could be maintained until $90 \mathrm{dpv}$, a booster dose on $28 \mathrm{dpv}$ could elicit a comparable humoral immune response as that of lapinized live CSFV vaccine until $180 \mathrm{dpv}$ under field conditions.

\section{Acknowledgements}

The authors thank the Dept. of Veterinary Microbiology, Madras Veterinary College, Chennai-7 and Tamil Nadu Veterinary and Animal Sciences University for all the facilities that supported this study

\section{References}

Aucouturier, J., L. Dupuis and V. Ganne, 2001. Adjuvants designed for Veterinary and human vaccines. Vaccine. 19 (17 - 19): $2666-2672$

Awate, S., L. A. Babiuk, and G. Mutwiri, 2013. Mechanisms of Action of Adjuvants. J. Frontiers in Immunology, 4: 114. 
Coffman, R. L., A. Sher, and R. A. Seder, 2010. Vaccine Adjuvants: Putting Innate Immunity to Work. J. Immunity, 33(4): $492-503$.

Dahle, J. and B. Liess, 1995. Assessment of safety and protective value of a cell culture modified strain " $C$ " vaccine of hog cholera/classical swine fever virus. Berl. Munch. Tierarztl. Wschr.108: 2025

Klinkenberg, D. R. J. Moormann, A. J. de Smit, A. Bouma and M. C. de Jong, 2002. Influence of maternal antibodies on efficacy of a subunit vaccine: Transmission of classical swine fever virus between pigs vaccinated at 2 weeks of age. Vaccine, 20: 3005-3013.

Liu, S., Y. Zhao, Hu, Q. Lv, C. Zhang, C. Zhao and S. Cui, 2011. A multiplex RT-PCR for rapid and simultaneous detection of porcine teschovirus, classical swine fever virus, and porcine reproductive and respiratory syndrome virus in clinical specimens. Journal of virological methods, 172(1): 88-92.

Mosca, F., E. Tritto, A. Muzzi, E. Monaci, F. Bagnoli, C. Iavarone, D. O'Hagan, R. Rappuoli and E. D. Gregorio, 2008. Molecular and cellular signatures of human vaccine adjuvants. Proceedings of the National Academy of Sciences, 105(30): 10501-10506

Nath, M. K., D. K. Sarma, B. C. Das, P. Deka, D. Kalita, J. B. Dutta, G. Mahato, S. Sarma and P. Roychoudhury, 2016. Evaluation of specific humoral immune response in pigs vaccinated with cell culture adapted classical swine fever vaccine. J. Vet. world, 9(3): 308-312

Park, M., S. Lee, R. Kim, M. Ko, J. Park, K. Lee, S. Kim, J. Choi, S. You, B. Kim and
J. Lee, 2016. Altered adjuvant of footand-mouth disease vaccine improves immune response and protection from virus challenge. Trials in Vaccinology, 5: 97-104.

Precausta, P., F. Kato and A. Brun, 1983. Swine fever. Immunisation of piglets. Comp. Immunol. Microbiol. Infect.Dis. 6:281-289

Sathyanarayanan. 2017. Evaluation of humoral and cell mediated immune responses in ruminants vaccinated with blue tongue vaccine (Ph.D., thesis submitted to TANUVAS).

Shahiwala, A. and M. M. Amiji, 2008. Enhanced mucosal and systemic immune response with squalane oilcontaining multiple emulsions upon intranasal and oral administration in mice. Journal of drug targeting, 16(4):302-310.

Tang, Y., J. Lu, P. Wu, Z. Liu, Z. Tian, G. Zha, H. Chen, Q. Wang, Q. Wang, F. Hou, S. M. Kang and J. Hou, 2014. Inactivated vaccine with adjuvants consisting of pattern recognition receptor agonists confers protection against avian influenza viruses in chickens. Veterinary Microbiology, 172(1-2): 120128

Terpstra C., R.Woortmeyer and S. J. Barteling, 1990. Development and properties of a cell culture produced vaccine for Hog cholera based on the Chinese strain. DtschTierarztl Wochenschr. 97: 77-79.

Terpstra, C. and M.J.M. Tielen, 1976. Antibody response against swine fever following vaccination with the C-strain virus. Z. Vet. Med. 23: 809-821.

\section{How to cite this article:}

Steffi Rajakumar, K.H., A. Ramesh, K.G. Tirumurugaan and Kumanan, K. 2018. Comparison of the Immunogenicity of an Inactivated Classical Swine Fever Virus Vaccine Employing a Local Isolate With the Live Lapinized Classical Swine Fever Virus Vaccine. Int.J.Curr.Microbiol.App.Sci. 7(10): 663-679. doi: https://doi.org/10.20546/ijcmas.2018.710.074 